\title{
Comparison of the Effect of Electrochemical Stimulation of the Medial Preoptic Area and the Hypothalamic Arcuate Nucleus upon LH Release in Ovariectomized and Proestrous Rats
}

\author{
Masazumi KAWAKAMI and TaKashi HIGUCHI \\ Department of Physiology \\ Yokohama City University School of Medicine, Yokohama 232, Japan
}

\begin{abstract}
The effect of electrochemical stimulation of the medial preoptic area (MPO) and the hypothalamic arcuate nucleus (ARC) on LH release was compared among longterm ovariectomized (OVX), ovariectomized rats treated with estradiol benzoate (OVX-E) and proestrous (PE) rats under pentobarbital anesthesia. The MPO stimulation failed to facilitate $\mathrm{LH}$ release in OVX rats whereas the stimulation increased serum LH levels in both OVX-E and PE rats. The ARC stimulation resulted in an elevation of serum LH in OVX and PE rats. The time course of serum LH elevation after the electrochemical stimulation of the MPO or the ARC was different between OVX-E and $\mathrm{PE}$ rats. In $\mathrm{PE}$ rats serum $\mathrm{LH}$ concentrations began to rise 20-30 min after the stimulation of the MPO or the ARC with peak values obtained around $70-90 \mathrm{~min}$. Serum LH started to elevate immediately with peak values in 10-20 min following the ARC stimulation in OVX rats and the MPO stimulation in OVX-E rats. The time courses for changes in serum LH concentrations following LH-RH administration were similar in OVX and PE rats. From these results, the lack of circadian rhythm in serum LH levels in long-term OVX rats may be due to the inability of the MPO to re- spond to the stimulus generated from circadian pacemaker(s). The cause of the delay observed between the stimulation of the MPO and LH rise in PE rats may not exist in the levels of neural transmission between the MPO and the ARC or the pituitary, but in the level of the mediobasal hypothalamus.
\end{abstract}

The medial preoptic area (MPO) of the brain is thought to be the center for the cyclic gonadotropin secretion which occurs at a fixed time of day under controlled lighting schedule in female rats (Everett and Sawyer, 1950; Halász and Gorski, 1967). By contrast, the arcuate nucleus (ARC) is thought to be one of the main sites responsible for tonic gonadotropin secretion and its activity is under the influence of the MPO (Halász and Gorski, 1967; Blake and Sawyer, 1974). Serum LH levels increase following ovariectomy and the elevated

Received January 10, 1980. levels which are thought to be tonic secretion, exhibit pulsatile patterns, but lack the circadian variation (Blake, 1974; Soper and Weick, 1977). It is necessary to administer estrogen to restore circadian rhythm in $\mathrm{LH}$ release in ovariectomized (OVX) rats (Caligaris et al., 1971; Legan and Karsch, 1975). Therefore it may be important to elucidate the function of the MPO in OVX rats for understanding the mechanism by which the cyclic gonadotropin surge occurs.

Electrical or electrochemical stimulation of the MPO or the ARC induce $\mathrm{LH}$ release and ovulation in cyclic rats in which spontaneous ovulation has been blocked with 
pentobarbital anesthesia (Everett and Radford, 1961; Kawakami et al., 1971 ; Turgeon and Barraclough, 1973). Although the precise mechanism of action of this procedure is not known, it is generally assumed that electrochemical or electrical stimulation of these areas induce luteinizing hormone-releasing hormone ( $\mathrm{LH}-\mathrm{RH})$ release by facilitating neural activity of the LHRH neurons (Terasawa and Sawyer, 1969; Kawakami et al., 1971; Van der School et al., 1978; Higuchi, 1978). There is still dispute on the causal relationship between the stimulated neural activity and the increase in serum LH. Terasawa and Sawyer (1969) observed an immediate increase in the multiunit discharge of the ARC lasting about $30 \mathrm{~min}$ after electrochemical stimulation of the MPO. But the serum concentration of $\mathrm{LH}$ does not rise until $30 \mathrm{~min}$ after electrochemical stimulation of the MPO (Kalra et al., 1971; Turgeon and Barraclough, 1973). The discrepancy between the rapid increase in neural activity and delayed elevation in serum LH levels remained to be explained.

In order to clarify the cause of the delayed increase in serum $\mathrm{LH}$ after the MPO stimulation and the function played by the MPO in OVX rats, we attempted to compare the effect of electrochemical stimulation of the MPO and the ARC on LH secretion and $\mathrm{LH}$ response to $\mathrm{LH}-\mathrm{RH}$ between in OVX and proestrous (PE) rats.

\section{Materials and Methods}

Female rats of Wistar strain (200-250 g body weight) were raised in a light controlled (lights on 0500 to $1900 \mathrm{hr}$ ), air-conditioned $\left(25 \pm 1^{\circ} \mathrm{C}\right)$ room and allowed free access to pelleted food (Orienal Kobo, Japan) and tap water. Vaginal smears were checked every morning and rats showing regular 4 day estrous cycles were used for the experiments. Ovariectomy was performed under ether anesthesia 1 to 2 months prior to the electrical stimulation study (OVX). A group of OVX rats were injected s.c. with estradiol benzoate (Teikoku Hormone Mfg. Co.
Japan) $72 \mathrm{hr}$ before the stimulation (OVX-E).

OVX, OVX-E or rats at the proestrous day (PE) were anesthetized with pentobarbital sodium (Abott, USA; $30 \mathrm{mg} / \mathrm{kg} \mathrm{BW}$ ) administered at 1330 to $1400 \mathrm{hr}$ and about $40-50 \mathrm{~min}$ after the pentobarbital injection blood samping was started at $10 \mathrm{~min}$ intervals for $60 \mathrm{~min}$. The animals were then electrochemically stimulated unilaterally through a concentric bipolar stainless steel electrode (inner diameter $0.13 \mathrm{~mm}$, outer diameter $0.4 \mathrm{~mm}$ ). An anodal current stimulus of 250 or $50 \mu \mathrm{A}$ was applied to the medial preoptic area (Anterior 8.2, Lateral 0.5, Depth 3.0-3.5; AlbeFessard et al., 1966) or the arcuate nucleus (Anterior 6.0, Lateral 0.1, Depth 1.0-1.5) for $30 \mathrm{sec}$ using electrical stimulator (Nihon Kohden MSE-3R). Sham stimulated rats were inserted with electrode into the MPO for $30 \mathrm{sec}$ without passing current. The site of stimulation was confirmed histologically from brain sections and distributed within an area $0.5-$ $0.6 \mathrm{~mm}$ from the aimed point.

Fifteen PE and 12 OVX rats were treated with either synthetic luteinizing hormone-releasing hormone (Sankyo Pharmaceutical Co., Japan, $0.5 \mu \mathrm{g} / \mathrm{m} l$ saline solution) or vehicle injected through intraatrial cannula.

Blood samples were taken through indwelling intra-atrial cannula implanted by $1000 \mathrm{hr}$ in the morning under ether anesthesia. One tenth $\mathrm{ml}$ of blood was taken at 10 min intervals and the same volume of physiological saline was replaced. Separated serum was kept at $-20^{\circ} \mathrm{C}$ until assayed for LH. Serum concentration of LH was determined by double antibody radioimmunoassay using antiovine LH serum (R-15; generous gift from Dr. G. D. Niswender, Colorado State University, USA) and NIAMDD rat $\mathrm{LH}$ radioimmunoassay kit (Niswender et al., 1968). The concentrations of LH in serum were expressed as ng NIH-LH-S1/ml serum. Statistical analysis was performed using one way analysis of variance or Student $t$ test.

\section{Results}

Effect of electrochemical stimulation of the MPO of the ARC on serum LH in OVX rats

Electrochemical stimulation of the MPO with current strength of $250 \mu \mathrm{A}$ for $30 \mathrm{sec}$ immediately inhibited pulsatile LH patterns for 20 to $\mathrm{min}$ thereafter (Fig. 1). Electrochemical stimulation of the MPO with smaller current strength $(50 \mu \mathrm{A}, 30 \mathrm{sec})$ also induced similar inhibition of the pulsatile LH secretion for 20 to $30 \mathrm{~min}$ (data not 

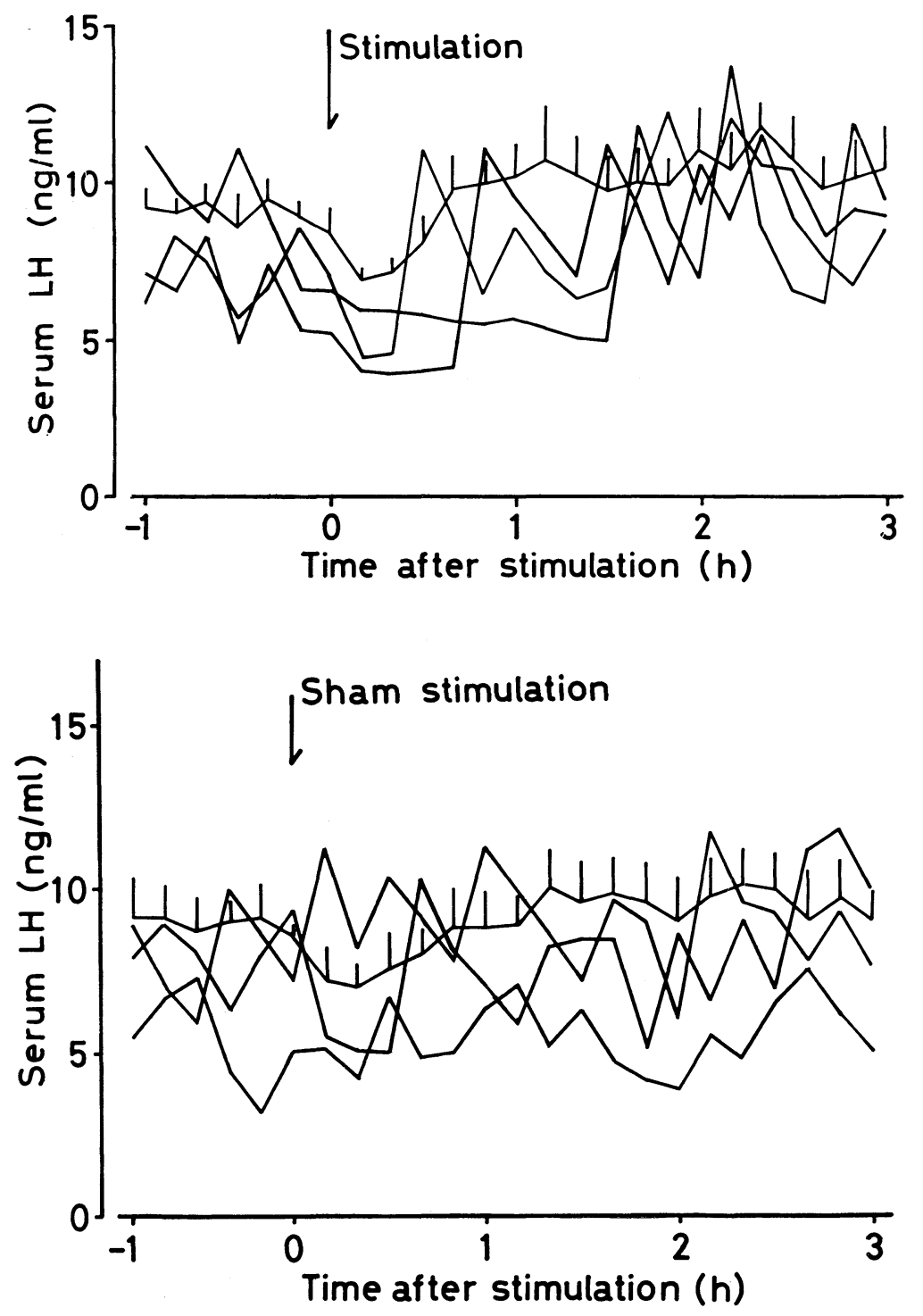

Fig. 1. Effect of electrochemical stimulation of the MPO on serum LH levels in three individual OVX rats. Line with vertical bars, which denote standard error of means, represents mean values of 13 rats.
Fig. 2. Effect of sham stimulation of the MPO on serum LH levels in three individual OVX rats. Line with vertical bars, which denote standard error of means, represents mean values of 6 rats. shown). None of the MPO stimulated rats (13 rats) exhibited an immediate increase of serum LH as those induced by the stimulation of the ARC. The inhibition of $\mathrm{LH}$ secretion was terminated by an abrupt resumption of the pulsatile secretory patterns which were of larger amplitude and longer duration compared with those observed during control period (Fig. 1).
Sham stimulated rats also exhibited inhibition of pulsatile patterns of serum $\mathrm{LH}$ concentration for $20-30 \mathrm{~min}$ in some animals (3 out of 6 rats as indicated individually in Fig. 2). Mean serum LH values at any time following MPO stimulation were not different for the stimulated and sham control groups.

Electrochemical stimulation of the ARC 
resulted in a rapid increase in serum $\mathrm{LH}$ with maximal levels occuring 10 to $30 \mathrm{~min}$ after the stimulation (Fig. 3). The serum LH returned to pre-stimulation levels after $70 \mathrm{~min}$, although some animals showed another small elevation ( 3 out of 7 rats) about 120 to $170 \mathrm{~min}$ after stimulation.

Effect of electrochemical stimulation of the MPO in OVX-E rats

In the OVX rats which had received estradiol benzoate (EB) injection of 2, 10 or $50 \mu \mathrm{g}, 3$ days prior to the stimulation, electrochemical stimulation of the MPO caused an elevation in serum $\mathrm{LH}$ with peak values appearing in $20-40 \mathrm{~min}$ after the stimulation in all the rats (5-6 rats in each group). More LH was released in the rats treated with $50 \mu \mathrm{g}$ of EB than in the rats treated with 2 or $10 \mu \mathrm{g}$ EB. This was quantified by comparing sum of LH values in each sample after subtraction of basal values before the stimulation $(121.8 \pm 13.2$ vs. $34.0 \pm 5.2 ; \mathrm{p}<0.001$, Student $t$ test). There was no apparent difference in the pattern of $\mathrm{LH}$ secretion after the MPO stimulation in the rats treaed with 2 and

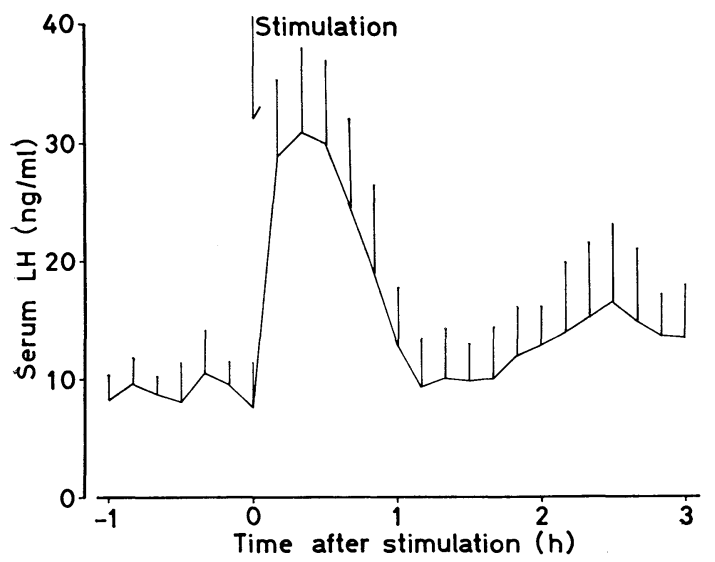

Fig. 3. Effect of electrochemical stimulation of the ARC on serum LH levels in OVX rats. Each point and vertical bar represent mean and standard error of means in 7 rats.
$10 \mu \mathrm{g}$ EB. The elevated serum LH concentrations declined more gradually in the rats receiving $50 \mu \mathrm{g}$ EB than those receiving 2 or $10 \mu \mathrm{g}$ EB (Fig. 4).

Effect of electrochemical stimulation of the $A R C$ or the $M P O$ in the PE rats

In $P E$ rats electrochemical stimulation of the MPO or the ARC with current strength of $250 \mu \mathrm{A}$ for $30 \mathrm{sec}$ increased serum levels of LH. LH values began to rise within $20-30 \mathrm{~min}$ and continued to increase until 70-90 min after stimulation. Then, the LH levels gradually declined but were still higher than the pre-stimulation levels $3 \mathrm{hr}$ after stimulation (Fig. 5). The time course of the elevation in serum $\mathrm{LH}$ concentrations in PE rats after MPO or ARC stimulation was different from those in OVX rats after ARC stimulation or in OVX-E rats after MPO stimulation. The peak values appeared later in PE rats than in OVX rats.

\section{$L H$ response to $L H-R H$ in $O V X$ and $P E$ rats}

In order to compare the pituitary res-

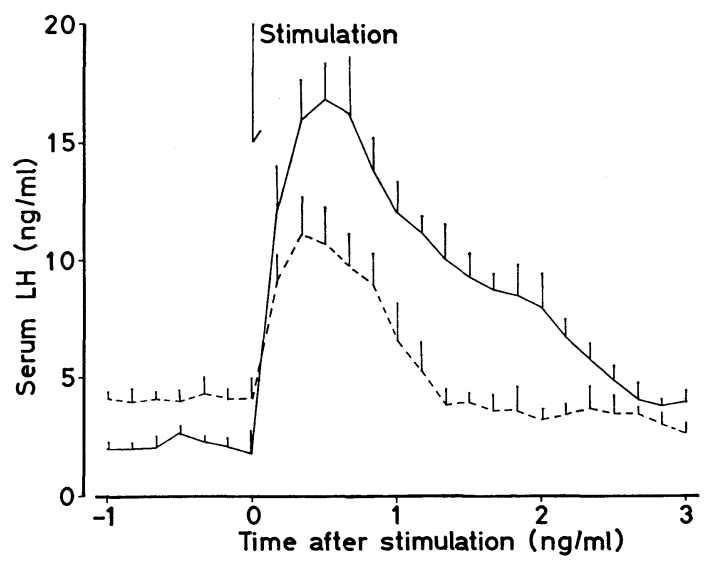

Fig. 4. Effect of electrochemical stimulation of the MPO in serum LH levels in OVX-E rats receiving $2 \mu \mathrm{g}$ (broken line) or $50 \mu \mathrm{g}$ (solid line) estradiol benzoate $72 \mathrm{hr}$ prior to the stimulation. Each point and vertical bar represent mean and standard error of means in 6 rats. 
ponsiveness to LH-RH in OVX and PE rats, synthetic LH-RH ( $500 \mathrm{ng} / \mathrm{kg} \mathrm{BW}$ ) was administered under anesthesia induced by pentobarbital between 1300 and $1345 \mathrm{hr}$ through cannula. Physiological saline $(0.2$ $\mathrm{ml}$ ) was administered to similarly treated control rats. Serum LH levels were determined immediately before and at $10 \mathrm{~min}$ intervals until $60 \mathrm{~min}$ after the LH-RH injection. As shown in Table 1 serum LH levels elevated within $10 \mathrm{~min}$ and peaked 10-20 min after LH-RH injection in both OVX and PE rats, but released amount of LH were significantly $(\mathrm{p}<0.01)$ greater in OVX than PE rats at every time when blood samples were taken during $60 \mathrm{~min}$ after LH$\mathrm{RH}$ injection. In control rats saline induced no apparent changes in serum LH concentrations.

\section{Discussion}

Electrochemical stimulation of the MPO failed to induce $\mathrm{LH}$ release in OVX rats without estrogen treatment as reported by Clemens et al. (972). This irresponsiveness of the MPO may closely relate to the absence of circadian variation in $\mathrm{LH}$ release in spayed rats (Blake, 1974; Soper and Weick, 1977). Estogen injection restored the ability of the MPO to activate $\mathrm{LH}$ release system. This fact is in accordance with the reappearance of phasic LH release which is related to the daily light-dark cycle in estrogen treated OVX rats (Caligaris et al., 1971; Legan and Karsch, 1975).
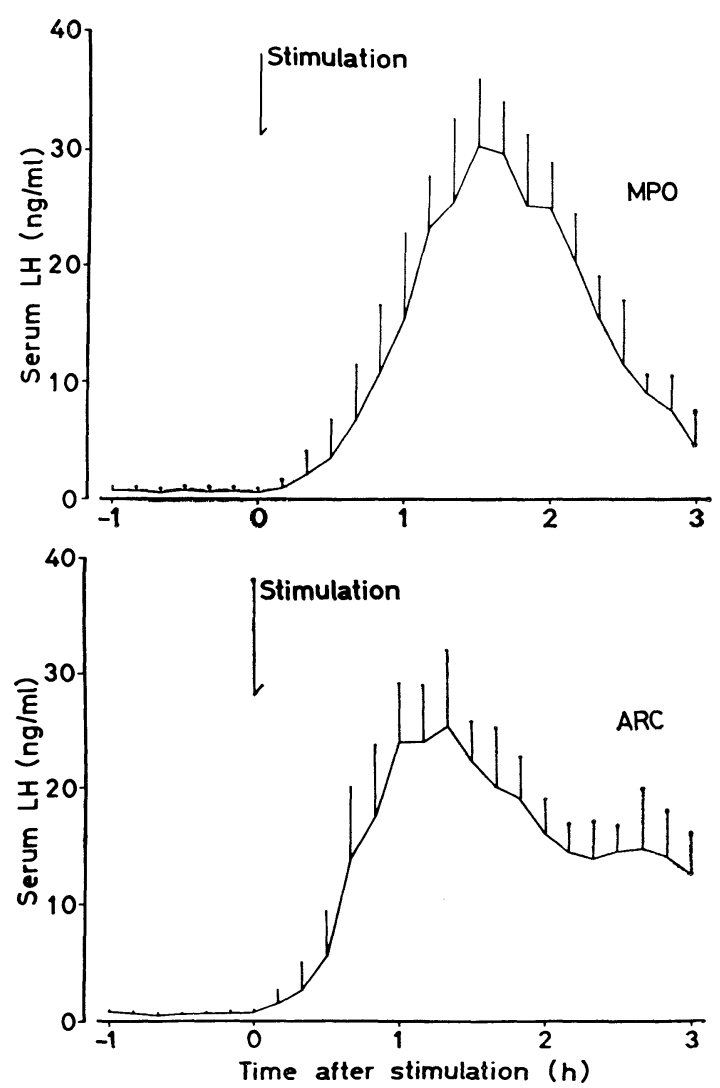

Fig. 5. Effect of electrochemical stimulation of the MPO (upper panel) or the ARC (lower panel) on serum LH levels in PE rats. Each point and vertical bar represent mean and standard error of means in 6 rads.

Table 1. Change in serum LH concentrations after LH-RH injection

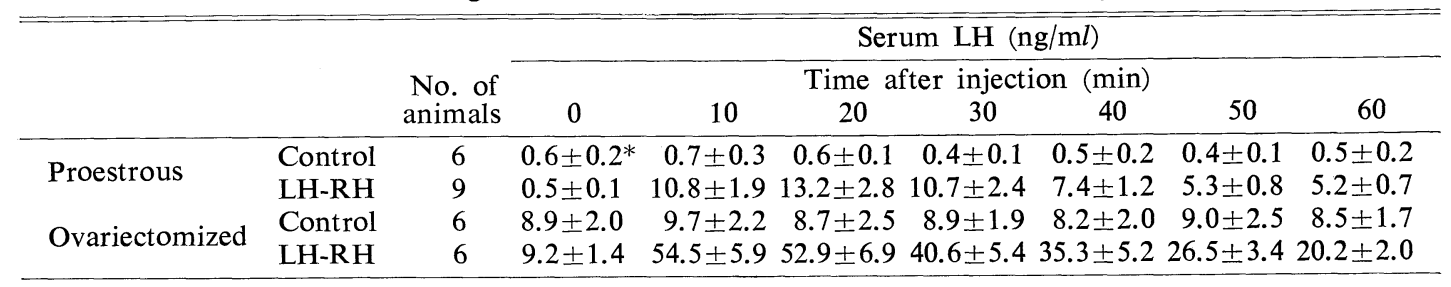

\footnotetext{
* mean \pm standard error of means
} 
The suprachiasmatic nucleus is an essential pacemaker of the circadian rhythm in cycle $\mathrm{LH}$ release as well as other functions that are influenced by light-dark cycles (Raisman and Brown-Grant, 1977; Moore and Eichler, 1972). The suprachias- matic nucleus and/or other pacemaker(s) may no longer operate in long-term OVX rats. However, circadian rhythms in other biological systems such as sleep-wakefulness (Kawakami et al., 1978), serum corticosterone rhythm (Hiroshige et al., 1973) are maintained following ovariectomy. Thus it is more possible that the stimulus originating from the neural pacemaker(s) reaches the MPO but this brain region then fails to activate $\mathrm{LH}-\mathrm{RH}$ release.

There are several possible reasons for the inability of the MPO stimulation to induce $\mathrm{LH}$ release in OVX rats which have not been pre-treated with estrogen. Low pituitary responsiveness to LH-RH is one of the possib lities. However, since the sensitivity of the pituitary was not lower in OVX than in PE rats, at least examined with the dose of LH-RH used $(500 \mathrm{ng} / \mathrm{kg}$ $\mathrm{BW}$ ), and that electrochemical stimulation of the ARC can induce LH release in OVX rats without estrogen treatment, we assume that MPO stimulation in spayed rats does not increase the amout of LH-RH reaching to anterior pituitary. The inability of the electrochemical stimulation of the MPO to induce $\mathrm{LH}$ release in OVX rats may be due to the higher threshold of MPO stimulation required to facilitate ARC neural activity in OVX rats (Kubo et al., 1975). Alternatively, extrahypothalamic area such as hippocampus may increase its inhibitory influence on responsiveness to the stimulation of the MPO (Kawakami et al., 1973) in the absence of ovarian steroids, and the inhibitory stimulus may counteract the facilitatory effect of the electrochemical stimulation of the MPO for LH-RH release. Furthermore, short-loop feedback action by the elevated serum $\mathrm{LH}$ on its secretion in
OVX rats may influence on the MPO sensitivity to electrochemical stimulation (Molitch et al., 1976). Another possibility is that there is less readily releasable LH-RH store in the hypothalamus in OVX rats as indicated by lower hypothalamic content of LH-RH in OVX rats than that in intact rats (Araki et al., 1975). But from our study, the mediobasal hypothalamic region seemed to have LH-RH store enough to activate $\mathrm{LH}$ release in OVX rats in response to electrochemical stimulation. Further studies are need to clarify which is the main cause of the inability of the MPO stimulation to release LH-RH in OVX rats.

Electrochemical stimulation of the MPO did not facilitate but rather suppressed pulsatile $\mathrm{LH}$ release in individual rats. However, mean LH levels following the stimulation were not different for the stimulated and sham control groups. This is probably because that resumed LH pulses following the inhibited period had higher amplitude and longer duration than those observed in prestimulation period. Different periods of inhibition and different time of reappearance of the pulsatile $\mathrm{LH}$ release in individual animals make mean $\mathrm{LH}$ levels equal to or greater than those in pre-stimulation period except 10 and $20 \mathrm{~min}$ after the stimulation. Moreover, since the inhibition of LH pulse we observed in some sham control rats, this inhibitory effect of the electrochemical stimulation on LH release may be explained by the general stress effect accompanied with the stimulation procedure.

Electrochemical stimulation of the MPO caused an elevation in serum $\mathrm{LH}$ in both $\mathrm{PE}$ and OVX-E rats, but the time course of the $\mathrm{LH}$ rise was not the same for the two groups. Serum LH level began to rise in $20 \mathrm{~min}$ and reached its peak $70-90$ min after the stimulation in the PE rats, in contrast with OVX-E rats in which it started to increase in $10 \mathrm{~min}$ with a peak 20-40 min after stimulation. These results 
confirmed relatively long time lag between the stimulation and $\mathrm{LH}$ rise in $\mathrm{PE}$ rats reported by others (Kalra et al., 1971; Turgeon and Barraclough, 1973). When the larger doses of estrogen were injected, the serum LH concentrations seem to remain at elevated levels for the longer period after the stimulation. But the peak values appeared uniformly within $20-40 \mathrm{~min}$ after stimulation irrespective of the estrogen dose. Moreover, ARC stimulation in OVX rats also induced a rapid increase in serum $\mathrm{LH}$, with maximal values occurring $10-30 \mathrm{~min}$ after stimulation. In these cases the time course of serum LH elevation following the electrochemical stimulation is similar to that following $\mathrm{LH}-\mathrm{RH}$ injection, indicating that rapid increase of $\mathrm{LH}-\mathrm{RH}$ secretion after the stimulation. Electrochemical stimulation in PE rats at the ARC as well as the MPO produced similar delay in increase of $\mathrm{LH}$. Thus the cause of this delay observed following MPO stimulation may not exist in neural transmission between the MPO and the ARC, but rather in the levels from the mediobasal hypothalamus including the ARC to the pituitary. As shown in Table $1 \mathrm{LH}$ $\mathrm{RH}$ injection caused a similar elevation in serum LH levels in both PE and OVX rats indicating that the difference in the time delay may not be due to the difference in the responsiveness of the pituitary. Since there is little elevation in serum LH until LH surge begins (Butcher et al., 1974) in spite of higher pituitary sensitivity to LHRH before the beginning of $\mathrm{LH}$ surge on proestrus (Cooper and McCann, 1975), some active mechanism inhibitory to LH-RH release may exist in the mediobasal hypothalamus.

\section{Acknowledgements}

We would like thank Drs. G. D. Niswender, Colorado State University, K. Wakabayashi, Gunma University and NIAMDD, USA for supplying radioimmunoassay materials. This research is supported by a grant from the Ministry of Education, Science and Culture of Japan ( $\$ 248118)$.

\section{References}

Albe-Fessard, D., F. Stutinsky and S. Libouban. Atlas stéréotaxique du diencéphale du rat blanc. Centre National de la Recherche Scientifique, Paris (1966).

Araki, S., M. Ferin, E. A. Zimmerman and R. L. Vande Wiele (1975). Endocrinology 96, 644.

Blake, C. A. (1974). ibid. 95, 813.

Blake, C. A. and C. H. Sawyer (1974). ibid. 94, 730.

Butcher, R. L., W. E. Collins and N. W. Fugo (1974). ibid. 94, 1704.

Caligaris, L., J. J. Astrada and S. Taleisnik (1971). ibid. 88, 810.

Clemens, J. A., C. J. Shaar and E. B. Smalstig (1972). Neuroendocrinology 10, 175.

Cooper, K. J. and S. M. McCann. Hypothalamic Hormones (edited by M. Motta, P. G. Crosignani and L. Martini). Academic Press, New York, p. 161 (1975).

Everett, J. W. and M. M. Radford (1961). Proc. Soc. Exp. Biol. Med. 108, 604.

Everett, J. W. and C. H. Sawyer (1950). Endocrinology 47, 198.

Halász, B. and R. A. Gorski (1967). ibid. 80, 608.

Higuchi, T. (1978). Folia Endocrinol. Japon. 54, 1163. (In Japanese).

Hiroshige, T., K. Abe, S. Wada and M. Kaneko (1973). Neuroendocrinology 11, 306.

Kalra, S. P., K. Ajika, L. Krulich, C. P. Fawcett, M. Quijada and S. M. McCann (1971). Endocrinology 88, 1150.

Kawakami, M., E. Terasawa, K. Seto and K. Wakabayashi (1971). Endocrinol. Japon. 18, 13.

Kawakami, M., E. Terasawa, F. Kimura and K. Wakabayashi (1973). Neuroendocrinology 12, 1.

Kawakami, M., S. Yamaoka and M. Matsushima. Environmental Endocrinology (edited by I. Assenmacher and D. S. Farner). Springer Verlag, Berlin, p. 132 (1978).

Kubo, K., R. A. Gorski and M. Kawakami (1975). Neuroendocrinology 18, 176.

Legan, S. J. and F. L. Karsch (1975). Endocrinology 96, 57

Molitch, M., M. Edmonds, E. E. Jones and W. E. Odell (1976). Am. J. Physiol. 230, 907. 
Moore, R. T. and V. E. Eichler (1974). Brain Res. 42, 201.

Niswender, G. D., A. R. Midgley, Jr., S. E. Monroe and L. E. Reichert, Jr. (1968). Proc. Soc. Exp. Biol. Med. 128. 807.

Raisman, G. and K. Brown-Grant (1977). Proc. $R$. Soc. Lond. B. 198, 297.

Soper, B. D. and R. F. Weick (1977). Neuroendo- crinology 24, 306.

Terasawa, E. and C. H. Sawyer (1973). Endocrinology 85, 143.

Turgeon, J. L. and C. A. Barraclough (1973). ibid. 92, 755 .

Van der Schoot, P., D. W. Lincoln and J. S. Clark (1978). J. Endocrinol. 79, 107. 